\title{
ABSOLUTELY COMPATIBLE PAIRS IN A VON NEUMANN ALGEBRA*
}

\author{
NABIN K. JANA ${ }^{\dagger}$, ANIL K. KARN ${ }^{\dagger}$, AND ANTONIO M. PERALTA ${ }^{\ddagger}$
}

Abstract. Let $a, b$ be elements in a unital $\mathrm{C}^{*}$-algebra with $0 \leq a, b \leq I$. The element $a$ is absolutely compatible with $b$ if

$$
|a-b|+|I-a-b|=I .
$$

In this note, some technical characterizations of absolutely compatible pairs in an arbitrary von Neumann algebra are found. These characterizations are applied to measure how far are two absolute compatible positive elements in the closed unit ball from being mutually orthogonal or commuting. In the case of 2 by 2 matrices, the results admit a geometric interpretation. Namely, non-commutative matrices of the form $a=\left(\begin{array}{cc}t & \alpha \\ \bar{\alpha} & 1-t\end{array}\right)$ and $b=\left(\begin{array}{cc}x & \beta \\ \beta & 1-x\end{array}\right)$ with $x, t \in(0,1) \backslash\left\{\frac{1}{2}\right\},|\alpha|^{2}<t(1-t)$ and $|\beta|^{2}<x(1-x)$, are absolutely compatible if, and only if, the corresponding point $\widetilde{b}=(x, \Re \mathrm{e}(\beta), \Im \mathrm{m}(\beta))$ in $\mathbb{R}^{3}$ lies in the ellipsoid

$$
\mathcal{E}_{a}=\left\{\bar{x} \in \mathbb{R}^{3}: d_{2}(\bar{x}, \widetilde{a})+d_{2}\left(\bar{x}, \widetilde{a^{\prime}}\right)=1\right\},
$$

where $d_{2}$ denotes the Euclidean distance in $\mathbb{R}^{3}$, and the elements $\widetilde{a}$ and $\widetilde{a^{\prime}}$ are $(t, \Re \mathrm{e}(\alpha), \Im \mathrm{m}(\alpha))$ and $(1-t,-\Re \mathrm{e}(\alpha),-\Im \mathrm{m}(\alpha))$, respectively. The description of absolutely compatible pairs of positive 2 by 2 matrices is applied to determine absolutely compatible pairs of positive elements in the closed unit ball of $\mathbb{M}_{n}$.

Key words. Absolute compatibility, Commutativity, $C^{*}$-algebra, von Neumann algebra, Projection, Partial isometry, Linear absolutely compatible preservers.

AMS subject classifications. 46L10, 46B 40, 46L05.

1. Introduction. The relation being orthogonal is a central notion of study in the setting of function algebras and in the non-commutative framework of general $\mathrm{C}^{*}$-algebras. Let us recall that elements $a$ and $b$ in a $\mathrm{C}^{*}$-algebra $A$ are called orthogonal ( $a \perp b$ in short) if $a b^{*}=b^{*} a=0$. Hermitian elements are orthogonal precisely when they have zero product. Following the standard notation, we shall write $|a|=\left(a^{*} a\right)^{\frac{1}{2}}$ for the absolute value of $a$.

Several attempts to establish a non-commutative version of the celebrated Kakutani's theorem [5], which characterizes those Banach lattices which are lattice isomorphic to the space $C(\Omega)$, of all continuous functions on a compact Hausdorff space $\Omega$, have been pursued in recent years (cf. [6, 7, 8]). As in many previous forerunners, like the representation theory published by Stone in [13] which characterizes $C(\Omega)$ in terms of order and its ring properties, a non-commutative Kakutani's theorem will necessarily rely on the notions of orthogonality, absolute value and order. Some discoveries have been found within this non-commutative program, for example, it is shown in [9, Proposition 4.9] that if $a$ is an arbitrary positive element in the closed unit ball, $\mathcal{B}_{A}$, of a unital $\mathrm{C}^{*}$-algebra $A$ and $p$ is a projection in $A$, then $|p-a|+|I-p-a|=I$ if and only if $a$ and $p$ commute. Furthermore, two positive elements $a$ and $b$ in $\mathcal{B}_{A}$ are orthogonal if, and only if,

${ }^{*}$ Received by the editors on October 25, 2018. Accepted for publication on October 18, 2019. Handling Editor: Torsten Ehrhardt. Corresponding Author: Antonio M. Peralta.

†School of Mathematical Sciences, National Institute of Science Education and Research, HBNI, Bhubaneswar, At \& Post Jatni, PIN - 752050, India (nabinjana@niser.ac.in, anilkarn@niser.ac.in).

${ }_{\ddagger}^{\ddagger}$ Departamento de Análisis Matemático, Facultad de Ciencias, Universidad de Granada, 18071 Granada, Spain (aperalta@ugr.es). Partial support by the Spanish Ministry of Science, Innovation and Universities (MICINN) and European Regional Development Fund project no. PGC2018-093332-B-I00 and Junta de Andalucía grant FQM375. 
$a+b \leq I$ and $|a-b|+|I-a-b|=I$. The second condition gives rise to a strictly weaker notion than the usual orthogonality. Accordingly to the notation in [9], given two elements $a, b \in A$ with $0 \leq a, b \leq I$, we shall say that $a$ is absolutely compatible with $b$ ( $a \triangle b$ in short) if

$$
|a-b|+|I-a-b|=I \text {. }
$$

Clearly, $a \perp b$ (with $0 \leq a, b \leq I$ ) implies that $a \triangle b$. It is shown in [9, Proposition 4.7] that $a$ is absolutely compatible with $b$ (with $0 \leq a, b \leq I$ ) if, and only if, $2 a \circ b=a+b-|a-b|$, where $a \circ b=\frac{1}{2}(a b+b a)$ is the usual Jordan product of $a$ and $b$. This notion is applied in [9] to introduce a spectral theory for absolute order unit spaces satisfying some specific conditions, which generalizes the spectral theory in von Neumann algebras.

In a recent contribution, we extend the notion of absolute compatibility to pairs of elements in the closed unit ball of an arbitrary (unital) $\mathrm{C}^{*}$-algebra $A$ via absolute values (see [3]). In this case, we introduce notions which are strictly weaker than range and domain orthogonality. Concretely, elements $a$ and $b$ in $\mathcal{B}_{A}$ are domain (respectively, range) absolutely compatible ( $a \triangle_{d} b$, respectively, $a \triangle_{r} b$, in short) if $|a|$ and $|b|$ (respectively, if $\left|a^{*}\right|$ and $\left|b^{*}\right|$ ) are absolutely compatible, that is,

$$
|| a|-| b||+|I-| a|-| b||=I
$$

(respectively, ||$\left.a^{*}|-| b^{*}||+|I-| a^{*}|-| b^{*}||=I\right)$. Finally, $a$ and $b$ are called absolutely compatible $(a \triangle b$ in short) if they are range and domain absolutely compatible.

One of the main results in [3] proves that every contractive linear operator $T$ between $\mathrm{C}^{*}$-algebras preserving domain absolutely compatible elements (i.e., $a \triangle_{d} b$ in $\mathcal{B}_{A} \Rightarrow T(a) \triangle_{d} T(b)$ ) or range absolutely compatible elements (i.e., $a \triangle_{r} b$ in $\mathcal{B}_{A} \Rightarrow T(a) \triangle_{r} T(b)$ ) is a triple homomorphism. Furthermore, a contractive linear operator between two $\mathrm{C}^{*}$-algebras preserves absolutely compatible elements (i.e., $a \triangle b$ in $\left.\mathcal{B}_{A} \Rightarrow T(a) \triangle T(b)\right)$ if, and only if, $T$ is a triple homomorphism. Having in mind the extensive literature on bounded linear operators between $\mathrm{C}^{*}$-algebras preserving (domain and/or range) orthogonality (for example, $[2,10,11,14,15])$, the results in $[3]$ inaugurate a new line to explore in the framework of preservers.

After characterizing triple homomorphisms as contractive linear operators between $\mathrm{C}^{*}$-algebras preserving absolutely compatible elements, it seems natural to explore how close or how far is a pair of absolutely compatible elements from being orthogonal. This comparison is the natural step in order to measure similarities and differences with linear orthogonality preservers. Absolute compatibility is not a mere technical workmanlike extension of previous notions, and will certainly play a role in the theory of preservers. This paper is aimed to throw some new light to our knowledge on absolutely compatible pairs of positive elements in the closed unit ball of a von Neumann algebra.

For each bounded linear operator $a$ acting on a complex Hilbert space $H$, the null projection (denoted by $n(a))$, and the range projection $(r(a)$ in short) are the orthogonal projections of $H$ onto the null space and the range space of $a$, which is the closure of $a(H)$, respectively. If $M$ is a von Neumann algebra in $B(H)$, then $r(a), n(a) \in M$ for each $a$ in $M$ (cf. [4, page 118], [12, 2.2.7]). It is known that if $a \in M$ is positive, the sequence $\left(a^{\frac{1}{n}}\right)_{n}$ converges to $r(a)$ in the weak* topology of $M$. Suppose $0 \leq a \leq I$ in $M$. The projection $s(a)=I-r(I-a)=w^{*}-\lim _{n} a^{n}$ will be called the support or peak projection of $a$. The symbol $e(a)$ will stand for the element $a-s(a)$. For example, let $a \in L^{\infty}[0,1]$ such that $a(t)=0$ on $[0,1 / 3], a(t)=1$ on $[2 / 3,1]$, and $0<a(t)<1$ on $(1 / 3,2 / 3)$. Then the range projections $r(a)=\chi_{(1 / 3,1]}, r(I-a)=\chi_{[0,2 / 3)}$, $n(a)=\chi_{[0,1 / 3]}$, while the peak projection $s(a)=I-r(I-a)=\chi_{[2 / 3,1]}$. 
The projections $s(a), r(e(a))$, and $n(a)$ are mutually orthogonal with

$$
s(a)+r(e(a))+n(a)=I,
$$

and every element in $M$ admits a matrix decomposition with respect to this system of projections.

Our first main result (see Theorem 2.2) is a technical characterization showing that two elements $a, b$ in a von Neumann algebra $M$ with $0 \leq a, b \leq I$, are absolutely compatible if, and only if, denoting by $p_{1}$ the range projection of $a \circ b$ in $M$, then $a$ and $b$ have matrix representations, say $a=\left(\begin{array}{cc}a_{11} & a_{12} \\ a_{12}^{*} & a_{22}\end{array}\right)$, and $b=\left(\begin{array}{cc}b_{11} & b_{12} \\ b_{12}^{*} & b_{22}\end{array}\right)$ with respect to the set $\left\{p_{1}, I-p_{1}=p_{2}\right\}$ (i.e., $a_{i j}=p_{i} a p_{j}$ and $\left.b_{i j}=p_{i} b p_{j}\right)$ satisfying the following properties:

(1) $a_{12}+b_{12}=0$;

(2) $\left|a_{12}^{*}\right|^{2}=\left(p_{1}-a_{11}\right)\left(p_{1}-b_{11}\right)$, (and hence, $\left.a_{11} b_{11}=b_{11} a_{11}\right)$;

(3) $\left|a_{12}\right|^{2}=a_{22} b_{22}=b_{22} a_{22}$;

(4) $a_{12}=a_{11} a_{12}+a_{12} a_{22}=b_{11} a_{12}+a_{12} b_{22}$.

This technical description admits some finer reformulations established in Theorems 2.6 and 2.10.

Furthermore, elements $0 \leq a, b \leq I$ are absolutely compatible if, and only if, there exist $0 \leq b_{1} \leq s(a)$, $0 \leq b_{2} \leq r(e(a))$, and $0 \leq b_{3} \leq n(a)$ such that $b_{2}$ is absolutely compatible with $e(a)$ and $b=b_{1}+b_{2}+b_{3}($ see Theorem 2.6).

These characterizations are subsequently applied to determine when a pair of absolutely compatible elements in a von Neumann algebra is a commuting pair. When particularized to the von Neumann algebra $\mathbb{M}_{n}$, of all $n \times n$ matrices with complex entries, the conclusions offer some interesting geometric interpretations. Commuting pairs of absolutely compatible positive elements in $\mathcal{B}_{\mathbb{M}_{2}}$ are described in Proposition 3.1. All possible pairs of absolutely compatible positive elements in $\mathcal{B}_{\mathbb{M}_{2}}$, in which one of the elements has non-zero peak projection or non-zero null projection are considered in Proposition 3.2. It is also shown that non-commuting $0 \leq a, b \leq I$ in $\mathbb{M}_{2}$ with $s(a)=n(a)=s(b)=n(b)=0$ are absolutely compatible if, and only if, $\operatorname{det}(a)>0$ and $\operatorname{det}(b)>0, \operatorname{trace}(a)=1=\operatorname{trace}(b)$, and $\operatorname{det}(a \circ b)=0$ (see Theorem 3.3). Finally, matrices of the form $a=\left(\begin{array}{cc}t & \alpha \\ \bar{\alpha} & 1-t\end{array}\right)$ and $b=\left(\begin{array}{cc}x & \beta \\ \bar{\beta} & 1-x\end{array}\right)$ with $x, t \in(0,1) \backslash\left\{\frac{1}{2}\right\},|\alpha|^{2}<t(1-t)$ and $|\beta|^{2}<x(1-x)$, are absolutely compatible if, and only if, the corresponding point $\widetilde{b}=(x, \Re \mathrm{e}(\beta), \Im \mathrm{m}(\beta))$ in $\mathbb{R}^{3}$ lies on the ellipsoid

$$
\mathcal{E}_{a}=\left\{\bar{x} \in \mathbb{R}^{3}: d_{2}(\bar{x}, \widetilde{a})+d_{2}\left(\bar{x}, \widetilde{a^{\prime}}\right)=1\right\},
$$

excluding the extremities of its major axis, where $d_{2}$ denotes the Euclidean distance in $\mathbb{R}^{3}, \widetilde{a}=(t, \Re \mathrm{e}(\alpha)$, $\Im \mathrm{m}(\alpha))$ and $\widetilde{a^{\prime}}=(1-t,-\Re \mathrm{e}(\alpha),-\Im \mathrm{m}(\alpha))$ (see Theorem 3.7).

In our final result we prove that absolutely compatible pairs of positive elements in $\mathcal{B}_{\mathbb{M}_{n}}$ can be represented as orthogonal sums of $2 \times 2$ matrices which are pairwise absolutely compatible (Theorem 3.9).

2. Characterization of positive absolute compatible elements. The notion of absolute compatibility was originally introduced in the setting of positive elements in the closed unit ball of a unital $\mathrm{C}^{*}$-algebra [9]. Among the results in the just quoted paper, we can find the following interesting characterization:

Proposition 2.1. [9, Propositions 4.5 and 4.7] Let $A$ be a unital $C^{*}$-algebra and let $0 \leq a, b \leq I$ be elements in $A$. Then the following statements are equivalent: 
(a) $a$ is absolutely compatible with $b$;

(b) $2 a \circ b=a+b-|a-b|$;

(c) $a \circ b,(I-a) \circ(I-b) \in A^{+}$and $(a \circ b)((I-a) \circ(I-b))=0$;

(d) $a \circ(I-b),(I-a) \circ b \in A^{+}$and $(a \circ(I-b))((I-a) \circ b)=0$;

(e) $a$ is absolutely compatible with $I-b$.

Throughout the remaining sections we shall focus on the set $[0, I]_{A}$ of all positive elements in the closed unit ball of a unital $\mathrm{C}^{*}$-algebra $A$.

Absolute compatibility in the commutative setting can be characterized in the following form: two positive functions $a, b$ in the closed unit ball of $C(K)$ are absolutely compatible if, and only if, $a(t) b(t)=0$ for all $t$ in $K$ with $a(t), b(t) \in[0,1)$ [3, Remark 2.2]. Inspired by this characterization, our first result is a matricial decomposition of an arbitrary couple of absolutely compatible positive elements in the closed unit ball of a von Neumann algebra.

TheOREM 2.2. Let $M$ be a von Neumann algebra, and let $a, b$ be elements in $[0, I]_{M}$. Then the following statements are equivalent:

(a) a is absolutely compatible with $b$;

(b) There exists a projection $p_{1}$ in $M$ so that $a$ and $b$ have matrix representations, say $a=\left(\begin{array}{ll}a_{11} & a_{12} \\ a_{12}^{*} & a_{22}\end{array}\right)$, and $b=\left(\begin{array}{ll}b_{11} & b_{12} \\ b_{12}^{*} & b_{22}\end{array}\right)$ with respect to the set $\left\{p_{1}, I-p_{1}=p_{2}\right\}$ (i.e., $a_{i j}=p_{i} a p_{j}$ and $\left.b_{i j}=p_{i} b p_{j}\right)$ satisfying:

(b.1) $p_{1}$ is the range projection of $a \circ b$, and $p_{1}=0$ if, and only if, $a \perp b$;

(b.2) $a_{12}+b_{12}=0$

(b.3) $\left|a_{12}^{*}\right|^{2}=\left(p_{1}-a_{11}\right)\left(p_{1}-b_{11}\right)$, (and hence, $\left.a_{11} b_{11}=b_{11} a_{11}\right)$;

(b.4) $\left|a_{12}\right|^{2}=a_{22} b_{22}=b_{22} a_{22}$;

(b.5) $a_{12}=a_{11} a_{12}+a_{12} a_{22}=b_{11} a_{12}+a_{12} b_{22}$.

Proof. Suppose first that $a$ is absolutely compatible with $b$. We deduce from Proposition 2.1 [9] that $a \circ b$ and $(I-a) \circ(I-b)$ are orthogonal elements in $[0, I]_{M}$, and thus,

$$
(a \circ b)^{2}=(a \circ b)(a+b-I)=(a+b-I)(a \circ b),
$$

which gives $r(a \circ b)(a+b-I)=(a+b-I) r(a \circ b)$, or equivalently,

$$
p_{1}(a+b)=(a+b) p_{1},
$$

where $p_{1}=r(a \circ b)$ is the range projection of $a \circ b$ in $M$.

We shall distinguish three cases.

Case 1. If $p_{1}=0$, we have $a \circ b=0$, and thus $a \perp b$, because in this case $0 \leq(I-a) \circ(I-b)=$ $I-a-b+a \circ b=I-a-b$, and hence, $0 \leq a+b \leq I$ (see Proposition 2.1), which combined with $a \triangle b$ gives $a \perp b[9$, Proposition 4.1].

Case 2. If $p_{1}=I$, we have $(I-a) \circ(I-b)=0$, and, by similar arguments to those given above, we get $(I-a) \perp(I-b)$. 
Case 3. Let us assume that $p_{1} \neq I, 0$. Let $a=\left(\begin{array}{cc}a_{11} & a_{12} \\ a_{12}^{*} & a_{22}\end{array}\right)$ and $b=\left(\begin{array}{cc}b_{11} & b_{12} \\ b_{12}^{*} & b_{22}\end{array}\right)$ be the matrix representations with respect to $\left\{p_{1}, I-p_{1}\right\}$. By applying the matrix representation in $(2.2)$ we get $a_{12}+b_{12}=$ 0.

Furthermore, if we write $a \circ b=\left(\begin{array}{cc}m_{0} & 0 \\ 0 & 0\end{array}\right)$ in our matrix representation, we deduce from (2.1) that $m_{0}^{2}=m_{0}\left(a_{11}+b_{11}-p_{1}\right)$, or equivalently, $m_{0}\left(a_{11}+b_{11}-p_{1}-m_{0}\right)=0$. It follows that $p_{1}\left(a_{11}+b_{11}-p_{1}-m_{0}\right)=$ $r\left(m_{0}\right)\left(a_{11}+b_{11}-p_{1}-m_{0}\right)=0$, that is,

$$
m_{0}=a_{11}+b_{11}-p_{1}
$$

Since $a$ is absolutely compatible with $b$, by Proposition 2.1, we also have $a \circ(I-b),(I-a) \circ b \in[0, I]_{M}$ with $(a \circ(I-b))((I-a) \circ b)=0$. That is, $(a-a \circ b)(b-a \circ b)=0$. Again, by computing the multiplication with respect to their matricial representations and applying (2.3), we get

$$
\begin{aligned}
\left(p_{1}-b_{11}\right)\left(p_{1}-a_{11}\right)-a_{12} a_{12}^{*} & =0 \\
-\left(p_{1}-b_{11}\right) a_{12}+a_{12} b_{22} & =0 \\
a_{12}^{*}\left(p_{1}-a_{11}\right)-a_{22} a_{12}^{*} & =0 \\
-a_{12}^{*} a_{12}+a_{22} b_{22} & =0
\end{aligned}
$$

These identities prove the desired statements.

We assume next the existence of a projection $p_{1} \in M$ such that $a$ and $b$ enjoy a matrix representation with respect to $\left\{p_{1}, 1-p_{1}\right\}$ satisfying (b.1)-to-(b.5). In this case, by $(b .2)$, the matrix representations of $a+b$ and $a-b$ with respect to $\left\{p_{1}, I-p_{1}\right\}$ are

$$
a+b=\left(\begin{array}{cc}
a_{11}+b_{11} & 0 \\
0 & a_{22}+b_{22}
\end{array}\right) \quad \text { and } \quad a-b=\left(\begin{array}{cc}
a_{11}-b_{11} & 2 a_{12} \\
2 a_{12}^{*} & a_{22}-b_{22}
\end{array}\right) \text {, }
$$

respectively.

Thus, the element $(a-b)^{2}$ writes in the form

$$
\left(\begin{array}{cc}
\left(a_{11}-b_{11}\right)^{2}+4 a_{12} a_{12}^{*} & 2\left(\left(a_{11}-b_{11}\right) a_{12}+a_{12}\left(a_{22}-b_{22}\right)\right) \\
2\left(\left(a_{11}-b_{11}\right) a_{12}+a_{12}\left(a_{22}-b_{22}\right)\right)^{*} & 4 a_{12}^{*} a_{12}+\left(a_{22}-b_{22}\right)^{2}
\end{array}\right) .
$$

Now, applying $(b .5),(b .3)$ and $(b .4)$ we deduce that

$$
\begin{gathered}
2\left(\left(a_{11}-b_{11}\right) a_{12}+a_{12}\left(a_{22}-b_{22}\right)\right)=0 \\
\left(a_{11}-b_{11}\right)^{2}+4 a_{12} a_{12}^{*}=\left(a_{11}-b_{11}\right)^{2}+4\left(p_{1}-a_{11}\right)\left(p_{1}-b_{11}\right)=\left(\left(p_{1}-a_{11}\right)+\left(p_{1}-b_{11}\right)\right)^{2},
\end{gathered}
$$

and

$$
4 a_{12}^{*} a_{12}+\left(a_{22}-b_{22}\right)^{2}=4 a_{22} b_{22}+\left(a_{22}-b_{22}\right)^{2}=\left(a_{22}+b_{22}\right)^{2} .
$$

We therefore have

$$
(a-b)^{2}=\left(\begin{array}{cc}
\left(\left(p_{1}-a_{11}\right)+\left(p_{1}-b_{11}\right)\right)^{2} & 0 \\
0 & \left(a_{22}+b_{22}\right)^{2}
\end{array}\right)
$$


and

$$
|a-b|=\left(\begin{array}{cc}
\left(p_{1}-a_{11}\right)+\left(p_{1}-b_{11}\right) & 0 \\
0 & a_{22}+b_{22}
\end{array}\right) .
$$

Next, again by (b.3) and (b.4) we also have

$$
\begin{aligned}
a b & =\left(\begin{array}{cc}
a_{11} b_{11}-a_{12} a_{12}^{*} & -a_{11} a_{12}+a_{12} b_{22} \\
a_{12}^{*} b_{11}-a_{22} a_{12}^{*} & -a_{12}^{*} a_{12}+a_{22} b_{22}
\end{array}\right) \\
& =\left(\begin{array}{cc}
a_{11}+b_{11}-p_{1} & -a_{11} a_{12}+a_{12} b_{22} \\
a_{12}^{*} b_{11}-a_{22} a_{12}^{*} & 0
\end{array}\right),
\end{aligned}
$$

and, by $(b .5), a \circ b=\left(\begin{array}{cc}a_{11}+b_{11}-p_{1} & 0 \\ 0 & 0\end{array}\right)$, which shows that $2(a \circ b)=a+b-|a-b|$, and Proposition 2.1 implies that $a$ is absolutely compatible with $b$.

Clearly, any two orthogonal elements commute. We have already commented that the same conclusion is not true for absolutely compatible positive elements in general. In the subsequent discussion, we shall find some finer characterizations for positive absolutely compatible pairs in a von Neumann algebra, and we shall isolate the reason for which a pair of positive absolutely compatible elements in the closed unit ball may not commute.

Proposition 2.3. Let $0 \leq a \leq I$ be an element in a von Neumann algebra $M$ with $n(a)=s(a)=0$. Then the following statements hold:

(i) $r(I-a)=I$;

(ii) If $0 \leq b \leq I$ is absolutely compatible with a such that $a b=b a$, then $b$ is a projection;

(iii) If $0 \leq b \leq I$ with $s(b)=n(b)=0$ satisfies that $a$ is absolutely compatible with $b$, then $a b \neq b a$;

(iv) If $x \in M$ such that $x^{*} a^{2} x=x^{*} x$, then $x=0$.

Proof. (i) Let $p=r(I-a)$. Since $(I-p) a=I-p$, and $n(a)=s(a)=0$, we get $0 \leq I-p \leq s(a)=0$, and thus, $p=I$.

(ii) Since $a b=b a$, and $a$ is absolutely compatible with $b$, Proposition 2.1 implies that $|a-b|=a+b-2 a b$. When squared we get

$$
\begin{aligned}
a^{2}+b^{2}-2 a b & =a^{2}+b^{2}+2 a b+4 a^{2} b^{2}-4 a b(a+b), \\
0 & =a b+a^{2} b^{2}-a b(a+b) .
\end{aligned}
$$

Thus, $a(I-a)\left(b-b^{2}\right)=0$. Since $n(a)=s(a)=0$, we have $r(I-a)=I=r(a)$, and hence, $b=b^{2}$.

(iii) It follows from $(i i)$.

(iv) Let us take $x \in M$ with $x^{*} a^{2} x=x^{*} x$. Since $x x^{*} a^{2} x x^{*}=x x^{*} x x^{*}$, we have $\left(x x^{*}\right)^{n} a^{2}\left(x x^{*}\right)^{m}=$ $\left(x x^{*}\right)^{n+m}$, for all $n, m \in \mathbb{N}$. Consequently, $z a^{2} w=z w$ for all $z, w$ in the von Neumann subalgebra generated by $x x^{*}$. Since the range projection $p=r\left(x x^{*}\right)$ lies in the latter von Neumann subalgebra, we deduce that $p a^{2} p=p$. Thus, $p\left(I-a^{2}\right) p=0$ and consequently $\left(I-a^{2}\right)^{\frac{1}{2}} p=0=\left(I-a^{2}\right) p=0$. Therefore, $a^{2} p=p a^{2}=p$. In particular, $p=p a=a p \leq s(a)=0$, because $n(a)=s(a)=0$. This proves that $x x^{*}=0$ or equivalently $x=0$.

Now, we prove a characterization for commuting pairs of positive elements in a von Neumann algebra. 
TheOREM 2.4. Let $M$ be a von Neumann algebra and let $0 \leq a \leq I$ in $M$. Then $0 \leq b \leq I$ commutes with $a$ if, and only if, there exist $0 \leq b_{1} \leq s(a), 0 \leq b_{2} \leq r(e(a))$ and $0 \leq b_{3} \leq n(a)$ such that $b_{2}$ commutes with $e(a)$, and $b=b_{1}+b_{2}+b_{3}$.

Proof. We assume first that $a b=b a$. We consider the commutative von Neumann subalgebra $N$ generated by $a, b$ and $I$. Let us observe that $n(a), e(a), s(a), n(b), e(b)$, and $s(b)$ all lie in $N \cong L^{\infty}(\mu)$. Taking $b_{1}:=s(a) b, b_{2}=r(e(a)) b$, and $b_{3}=n(a) b$, it is not hard to check that these elements satisfy the desired properties.

The reciprocal implication can be easily checked in a routine way.

Lemma 2.5. Let $0 \leq a, b \leq I$ in a von Neumann algebra $M$. Suppose $p$ is a projection in $M$. Then the following statements hold:

(a) If $a, b \in p M p$, then $a \triangle b$ in $M$ if, and only if, $a \triangle b$ in $p M p$;

(b) If $a, b \in p M p \oplus(I-p) M(I-p)$, then $a \triangle b$ in $M$ if, and only if, pap $\triangle p b p$ in $p M p$ and $(I-p) a(I-$ $p) \triangle(I-p) b(I-p)$ in $(I-p) M(I-p)$.

Proof. (a) Suppose $a, b \in p M p$. By orthogonality,

$$
|a-b|+|1-a-b|=|a-b|+|(I-p)+p-a-b|=|a-b|+(I-p)+|p-a-b|
$$

with $|a-b|,|p-a-b| \in p M p$. Therefore, $1=|a-b|+|1-a-b|$ if, and only if, $p=|a-b|+|p-a-b|$.

The proof of $(b)$ follows by similar arguments.

In Theorem 2.4, we characterize commuting pairs of positive elements in a von Neumann algebra. Next we consider pairs of positive absolutely compatible elements.

TheOrem 2.6. Let $M$ be a von Neumann algebra and let $0 \leq a \leq I$ be an element in $M$. Then $0 \leq b \leq I$ in $M$ is absolutely compatible with a if, and only if, there exist $0 \leq b_{1} \leq s(a), 0 \leq b_{2} \leq r(e(a))$, and $0 \leq b_{3} \leq n(a)$ such that $b_{2}$ is absolutely compatible with $e(a)$ and $b=b_{1}+b_{2}+b_{3}$.

Proof. Assume first that $a$ is absolutely compatible with $b$. By Proposition 2.1 we know that

$$
|a-b|=a+b-2 a \circ b,
$$

with $a \circ b \geq 0$. Consider the matrix representations of $a$ and $b$ with respect to $\{s(a), r(e(a)), n(a)\}$ :

$$
a=\left(\begin{array}{ccc}
s(a) & 0 & 0 \\
0 & e(a) & 0 \\
0 & 0 & 0
\end{array}\right) \quad \text { and } \quad b=\left(\begin{array}{ccc}
b_{11} & b_{12} & b_{13} \\
b_{12}^{*} & b_{22} & b_{23} \\
b_{13}^{*} & b_{23}^{*} & b_{33}
\end{array}\right)
$$

Now, by matrix multiplication, we get

$$
2 a \circ b=a b+b a=\left(\begin{array}{ccc}
2 b_{11} & b_{12}+b_{12} e(a) & b_{13} \\
e(a) b_{12}^{*}+b_{12}^{*} & 2 e(a) \circ b_{22} & e(a) b_{23} \\
b_{13}^{*} & b_{23}^{*} e(a) & 0
\end{array}\right) .
$$

Since $a \circ b \geq 0$ (see Proposition 2.1), we conclude that $b_{13}=0$ and $e(a) b_{23}=0$, and then $b_{23}=$ $r(e(a)) b_{23}=0$. Now, by $(2.4)$, we get

$$
|a-b|=a+b-2 a \circ b=\left(\begin{array}{ccc}
s(a)-b_{11} & -b_{12} e(a) & 0 \\
-e(a) b_{12}^{*} & e(a)+b_{22}-2 e(a) \circ b_{22} & 0 \\
0 & 0 & b_{33}
\end{array}\right)
$$


which assures that $|a-b|^{2}=\left(x_{i j}\right)$, where

$$
\begin{aligned}
& x_{11}=\left(s(a)-b_{11}\right)^{2}+b_{12} e(a)^{2} b_{12}^{*}, \\
& x_{12}=-\left(s(a)-b_{11}\right) b_{12} e(a)-b_{12} e(a)\left(e(a)+b_{22}-e(a) \circ b_{22}\right), \\
& x_{13}=0=x_{23}, \\
& x_{22}=e(a) b_{12}^{*} b_{12} e(a)+\left(e(a)+b_{22}-2 e(a) \circ b_{22}\right)^{2}, \\
& x_{33}=b_{33}^{2}
\end{aligned}
$$

and $x_{j i}=x_{i j}^{*}$. Similarly, we can show that $(a-b)^{2}=\left(y_{i j}\right)$, where

$$
\begin{aligned}
& y_{11}=\left(s(a)-b_{11}\right)^{2}+b_{12} b_{12}^{*}, \\
& y_{12}=-\left(s(a)-b_{11}\right) b_{12}-b_{12}\left(e(a)-b_{22}\right), \\
& y_{13}=0=y_{23}, \\
& y_{22}=b_{12}^{*} b_{12}+\left(e(a)-b_{22}\right)^{2}, \\
& y_{33}=b_{33}^{2}
\end{aligned}
$$

and $y_{j i}=y_{i j}^{*}$. We apply now that $|a-b|^{2}=(a-b)^{2}$, and hence, $x_{i j}=y_{i j}$ for $1 \leq i, j \leq 3$. The equation $x_{11}=y_{11}$ gives $b_{12} e(a)^{2} b_{12}^{*}=b_{12} b_{12}^{*}$, and thus, by Proposition 2.3(iv), we have $b_{12}^{*}=0$ so that $b_{12}=0$.

Now, comparing $x_{22}=y_{22}$, we get

$$
\left(e(a)+b_{22}-2 e(a) \circ b_{22}\right)^{2}=\left(e(a)-b_{22}\right)^{2} .
$$

Furthermore, since $|a-b| \geq 0$, we have $e(a)+b_{22}-2 e(a) \circ b_{22} \geq 0$, and hence,

$$
\left|e(a)-b_{22}\right|=e(a)+b_{22}-2 e(a) \circ b_{22} .
$$

Thus, $e(a)$ is absolutely compatible with $b_{22}$ (compare Proposition 2.1). We have therefore shown that $b=b_{11}+b_{22}+b_{33}$, where $b_{11} \in[0, s(a)], b_{22} \in[0, r(e(a))], b_{33} \in[0, n(a)]$, and $b_{22}$ is absolutely compatible with $e(a)$.

Conversely, let us assume that there exist $b_{1} \in[0, s(a)], b_{2} \in[0, r(e(a))]$ and $b_{3} \in[0, n(a)]$ with $b_{2}$ absolutely compatible with $e(a)$ such that $b=b_{1}+b_{2}+b_{3}$. We shall show that $a$ is absolutely compatible with $b$. Having in mind that the projections in $\{s(a), r(e(a)), n(a)\}$ are mutually orthogonal, we deduce that

$$
\begin{aligned}
|a-b| & =\left|s(a)+e(a)-b_{1}-b_{2}-b_{3}\right| \\
& =\left|\left(s(a)-b_{1}\right)+\left(e(a)-b_{2}\right)+\left(0-b_{3}\right)\right| \\
& =\left|s(a)-b_{1}\right|+\left|e(a)-b_{2}\right|+\left|0-b_{3}\right| \\
& =\left(s(a)-b_{1}\right)+\left|e(a)-b_{2}\right|+b_{3},
\end{aligned}
$$

and

$$
\begin{aligned}
|I-a-b| & =\left|I-s(a)-e(a)-b_{1}-b_{2}-b_{3}\right| \\
& =\left|\left(0-b_{1}\right)+\left(r(e(a))-e(a)-b_{2}\right)+\left(n(a)-b_{3}\right)\right| \\
& =\left|0-b_{1}\right|+\left|r(e(a))-e(a)-b_{2}\right|+\left|n(a)-b_{3}\right| \\
& =b_{1}+\left|r(e(a))-e(a)-b_{2}\right|+\left(n(a)-b_{3}\right) .
\end{aligned}
$$


Adding these terms, we get

$$
|a-b|+|I-a-b|=s(a)+\left|e(a)-b_{2}\right|+\left|r(e(a))-e(a)-b_{2}\right|+n(a) .
$$

Since $e(a)$ is absolutely compatible with $b_{2}$, Lemma 2.5(a) implies that $\left|e(a)-b_{2}\right|+\left|r(e(a))-e(a)-b_{2}\right|=$ $r(e(a))$, and thus, $|a-b|+|I-a-b|=I$, as desired.

REMARK 2.7. Let $M$ be a von Neumann algebra and let $a, b \in[0, I]$. If $a$ is absolutely compatible with $b$, then every distinct pair of elements in the set $\left\{s(a), e(a), n(a), b_{1}, b_{2}, b_{3}\right\}$ is absolutely compatible. It is also true that each element in the set $\{s(a), e(a), r(e(a)), n(a), a, a+n(a)\}$ is absolutely compatible with every element in the set $\left\{b_{1}, b_{2}, b_{3}, b_{1}+b_{2}, b_{1}+b_{3}, b_{2}+b_{3}, b\right\}$.

REMARK 2.8. Let $M$ be a von Neumann algebra, and let $a, b \in[0, I]$. If $a \triangle b$ then by Theorem 2.6 , $b=b_{1}+b_{2}+b_{3}$ such that $b_{1} \in[0, s(a)], b_{2} \in[0, r(e(a))], b_{3} \in[0, n(a)]$, and $b_{2} \Delta e(a)$. If in addition, $n(b)=s(b)=0$, then $I=r(b)=r\left(b_{1}\right)+r\left(b_{2}\right)+r\left(b_{3}\right)$. It follows that $r\left(b_{1}\right)=s(a), r\left(b_{2}\right)=r(e(a))$ and $r\left(b_{3}\right)=n(a)$. Now $b_{1}=0$ if, and only if, $s(a)=0 ; b_{2}=0$ if, and only if, $e(a)=0$; and $b_{3}=0$ if, and only if, $n(a)=0$.

We recall that for a projection $p$ and $0 \leq a \leq I$ in a $\mathrm{C}^{*}$-algebra $A$, we have $p a=a p$ if, and only if, $p$ is absolutely compatible with $a$ (see [9, Proposition 4.9]). Thus, it follows from Proposition 2.3( $i i$ ) that the next result is an assimilation of Theorems 2.4 and 2.6.

Corollary 2.9. Let $0 \leq a, b \leq I$ be elements in a von Neumann algebra $M$. Then $a b=b a$ and $a \triangle b$ hold if, and only if, the following statements are true:

(1) $b=b_{1}+b_{2}+b_{3}$ with $0 \leq b_{1} \leq s(a), 0 \leq b_{2} \leq r(e(a))$ and $0 \leq b_{3} \leq n(a)$;

(2) $b_{2}$ is a projection with $b_{2} e(a)=e(a) b_{2}$.

Let $a$ and $b$ be positive elements in the closed unit ball of a von Neumann algebra $M$. Suppose $a$ is absolutely compatible with $b$. By Theorem 2.6, there exist $b_{1} \in[0, s(a)], b_{2} \in[0, r(e(a))]$ and $b_{3} \in[0, n(a)]$ with $e(a)$ absolutely compatible with $b_{2}$ such that $b=b_{1}+b_{2}+b_{3}$. The elements $b_{2}$ and $e(a)$ form an absolutely compatible pair in the closed unit ball of the von Neumann algebra $r(e(a)) M r(e(a))$ (Lemma 2.5). We define the relative kernel of $b_{2}$ with respect to $e(a)$ as the projection $n_{1}\left(b_{2}\right)=r(e(a))-s\left(b_{2}\right)-r\left(e\left(b_{2}\right)\right)$.

We further sharpen the conclusion in Theorem 2.6.

THEOREM 2.10. Let $a$ and $b$ be elements in a von Neumann algebra $M$ such that $0 \leq a, b \leq I$. Then $a$ and $b$ are absolutely compatible if, and only if, there exist positive elements $a_{1}, a_{2}, a_{3}, b_{1}, b_{2}, b_{3}$ in $M$ and mutually orthogonal (possibly zero) projections $s_{1}, s_{2}, r, n_{1}$ and $n_{2}$ in $M$ satisfying:

(a) $s_{1}+s_{2}+r+n_{1}+n_{2}=I$

(b) $b_{1} \leq s_{1}, a_{1} \leq s_{2}, a_{2} \leq r=r\left(b_{2}\right), b_{3} \leq n_{1}, a_{3} \leq n_{2}$ and $a_{2}$ is absolutely compatible with $b_{2}$;

(c) $a=s_{1}+a_{1}+a_{2}+a_{3}$ and $b=b_{1}+s_{2}+b_{2}+b_{3}$.

Proof. Suppose first that $a$ and $b$ are absolutely compatible. By Theorem 2.6, there exist $b_{1} \in[0, s(a)]$, $b_{2} \in[0, r(e(a))]$ and $b_{3} \in[0, n(a)]$ with $e(a)$ absolutely compatible with $b_{2}$ such that $b=b_{1}+b_{2}+b_{3}$. Let $n_{1}\left(b_{2}\right)$ denote the relative kernel of $b_{2}$ with respect to $e(a)$. A new application of Theorem 2.6 to the absolutely compatible pair $b_{2}, e(a)$ in the von Neumann algebra $r(e(a)) M r(e(a))$, proves the existence of $a_{1} \in\left[0, s\left(b_{2}\right)\right], a_{2} \in\left[0, r\left(e\left(b_{2}\right)\right)\right]$ and $a_{3} \in\left[0, n_{1}\left(b_{2}\right)\right]$ with $a_{2}$ absolutely compatible with $e\left(b_{2}\right)$ such that $e(a)=a_{1}+a_{2}+a_{3}$, and thus, $a=s(a)+a_{1}+a_{2}+a_{3}$ and $b=b_{1}+s\left(b_{2}\right)+e\left(b_{2}\right)+b_{3}$. 
Conversely, assume that there exist positive elements $a_{1}, a_{2}, a_{3}, b_{1}, b_{2}, b_{3}$ in $M$ and mutually orthogonal (possibly zero) projections $s_{1}, s_{2}, r, n_{1}$ and $n_{2}$ in $M$ satisfying $(a),(b)$ and $(c)$. Then we have

$$
\begin{aligned}
|a-b| & =\left|\left(s_{1}+a_{1}+a_{2}+a_{3}\right)-\left(b_{1}+s_{2}+b_{2}+b_{3}\right)\right| \\
& =\left|\left(s_{1}-b_{1}\right)+\left(a_{1}-s_{2}\right)+\left(a_{2}-b_{2}\right)+a_{3}-b_{3}\right| \\
& =\left|s_{1}-b_{1}\right|+\left|a_{1}-s_{2}\right|+\left|a_{2}-b_{2}\right|+\left|a_{3}\right|+\left|-b_{3}\right| \\
& =\left(s_{1}-b_{1}\right)+\left(s_{2}-a_{2}\right)+\left|a_{2}-e\left(b_{2}\right)\right|+a_{3}+b_{3}
\end{aligned}
$$

and

$$
\begin{aligned}
|I-a-b| & =\left|I-\left(s_{1}+a_{1}+a_{2}+a_{3}\right)-\left(b_{1}+s_{2}+b_{2}+b_{3}\right)\right| \\
& =\left|-b_{1}-a_{1}+\left(r-a_{2}-b_{2}\right)+\left(n_{2}-a_{3}\right)+\left(n_{1}-b_{3}\right)\right| \\
& =\left|-b_{1}\right|+\left|-a_{1}\right|+\left|r-a_{2}-b_{2}\right|+\left|n_{2}-a_{3}\right|+\left|n_{1}-b_{3}\right| \\
& =b_{1}+a_{1}+\left|r-a_{2}-b_{2}\right|+\left(n_{2}-a_{3}\right)+\left(n_{1}-b_{3}\right) .
\end{aligned}
$$

Having in mind that $a_{2}$ is absolutely compatible with $b_{2}$, Lemma 2.5 yields that

$$
\left|a_{2}-b_{2}\right|+\left|r-a_{2}-b_{2}\right|=r .
$$

Thus, by adding the last three equations, we get $|a-b|+|I-a-b|=I$, which concludes the proof.

Let us observe that some of the projections, and consequently, some of the corresponding elements in Theorem 2.10 may be zero.

Corollary 2.11. Let $a$ and $b$ be elements in a von Neumann algebra $M$ such that $0 \leq a, b \leq I$. Suppose that $a$ is absolutely compatible with $b$. Then $a b=b a$ if, and only if, the element $b_{2}$ given by Theorem 2.6 is a projection.

Proof. If $a$ and $b$ commute, let $N$ denote the commutative von Neumann subalgebra of $M$ generated by $a, b$ and $I$. By identifying $N$ with a $C(K)$ space and having in mind that $a \triangle b$, we deduce from [3, Remark 2.2 ] that $b_{2}$ must be a projection in $N$ and hence in $M$.

Suppose now that $b_{2}$ is a projection, and hence, $e\left(b_{2}\right)=0$. By applying Theorem 2.10 we deduce that $a_{2}=0$, and hence, $a=s(a)+a_{1}+a_{3}$ and $b=b_{1}+s\left(b_{2}\right)+b_{3}$ satisfy the conclusion of the just commented theorem. It can be checked from the conclusion of this Theorem 2.10 that $a b=b a$.

We resume our previous conclusion in a more schematic form.

REMARK 2.12. Let $M$ be a von Neumann algebra and let $0 \leq a, b \leq I$ such that $a \triangle b$.

(1) If $a$ and $b$ commute, then there exists a set of mutually orthogonal projections $\left\{s(a), b_{2}, n_{1}\left(b_{2}\right), n(a)\right\}$ in $M$ whose sum is $I$ such that $a$ and $b$ have the following matrix representations with respect to this system:

$$
a=\left(\begin{array}{cccc}
s(a) & 0 & 0 & 0 \\
0 & a_{1} & 0 & 0 \\
0 & 0 & a_{3} & 0 \\
0 & 0 & 0 & 0
\end{array}\right) \quad \text { and } \quad b=\left(\begin{array}{cccc}
b_{1} & 0 & 0 & 0 \\
0 & b_{2} & 0 & 0 \\
0 & 0 & 0 & 0 \\
0 & 0 & 0 & b_{3}
\end{array}\right) \text {. }
$$

Some of these projections may be zero. 
(2) If $a$ and $b$ do not commute, then there exists a set of mutually orthogonal projections $\left\{s(a), s\left(b_{2}\right), q_{1}, q_{2}\right.$, $\left.n_{1}\left(b_{2}\right), n(a)\right\}$ in $M$ whose sum is $I$ such that $a$ and $b$ have the following matrix representations with respect to this system:

$$
a=\left(\begin{array}{cccccc}
s(a) & 0 & 0 & 0 & 0 & 0 \\
0 & a_{1} & 0 & 0 & 0 & 0 \\
0 & 0 & a_{11} & a_{12} & 0 & 0 \\
0 & 0 & a_{12}^{*} & a_{22} & 0 & 0 \\
0 & 0 & 0 & 0 & a_{3} & 0 \\
0 & 0 & 0 & 0 & 0 & 0
\end{array}\right), \quad b=\left(\begin{array}{cccccc}
b_{1} & 0 & 0 & 0 & 0 & 0 \\
0 & s\left(b_{2}\right) & 0 & 0 & 0 & 0 \\
0 & 0 & b_{11} & -a_{12} & 0 & 0 \\
0 & 0 & -a_{12}^{*} & b_{22} & 0 & 0 \\
0 & 0 & 0 & 0 & 0 & 0 \\
0 & 0 & 0 & 0 & 0 & b_{3}
\end{array}\right) .
$$

Some of these projections may be zero. However, $q_{1}$ and $q_{2}$ can not be zero. Similarly, $a_{12} \neq 0$. Moreover, $a_{11}$ commutes with $b_{11}$ and $a_{22}$ commutes with $b_{22}$.

3. Absolute compatibility in the case of matrices. In this section, we shall particularize the main conclusions in Section 2 to the case of matrix algebras. Note that, in a general von Neumann algebra $M$, 0 and $I$ are absolutely compatible with every element $a \in[0, I]_{M}$. Thus, in order to describe absolutely compatible elements in $M$ it suffices to discuss the absolutely compatible pairs in $[0, I]_{M} \backslash\{0, I\}$.

3.1. Absolute compatibility in $\mathbb{M}_{2}$. In this subsection, we discuss the case of 2 by 2 matrices due to its special importance. Commuting pairs of absolutely compatible 2 by 2 matrices are described in the next result.

Proposition 3.1. Let $0 \leq a, b \leq I$ in $\mathbb{M}_{2}$ such that $a \triangle b$ and $a b=b a$. Then the following statements hold:

(a) If $n(a)=s(a)=0$, then there exist $\alpha, \beta \in(0,1)$ in $\mathbb{R}$ and a rank one projection $p \in \mathbb{M}_{2}$ such that $b=p$ and $a=\alpha p+\beta(I-p)$;

(b) If $n(a) \neq 0$ or $s(a) \neq 0$, then one of the next statements holds:

(b.1) If e $(a)=0$, then there exist $\alpha, \beta \in[0,1]$ in $\mathbb{R}$ and a rank one projection $p \in \mathbb{M}_{2}$ such that $\alpha+\beta>0$ and $a=p$ and $b=\alpha p+\beta(I-p)$;

(b.2) If $s(a), e(a) \neq 0$, then there exist $\alpha, \beta \in[0,1]$ in $\mathbb{R}$ and a rank one projection $p \in \mathbb{M}_{2}$ such that $a=p+\alpha(I-p)$ and $b=\beta p+(I-p)$ with $\alpha \neq 0$ or $a=p+\alpha(I-p)$ and $b=\beta p$ with $\alpha, \beta \neq 0$;

(b.3) If $n(a), e(a) \neq 0$, then there exist $\alpha, \beta \in[0,1]$ in $\mathbb{R}$ and a rank one projection $p \in \mathbb{M}_{2}$ such that $a=\alpha p$ and $b=p+\beta(I-p)$ with $\alpha \neq 0$ or $a=\alpha p$ and $b=\beta(I-p)$ with $\alpha, \beta \neq 0$.

Proof. The statement is a straight consequence of Remark 2.12(1) and the fact that in $\mathbb{M}_{2}$ we can find at most two orthogonal rank one projections. It can be also obtained from Corollary 2.11 or from the fact that there exists a rank one projection $p$ and $\alpha, \beta$ in $[0,1]$ such that $a=\alpha p+\beta(I-p)$.

In our next result, we study absolutely compatible pairs in which one of the elements has non-zero null or peak projection.

Proposition 3.2. Let $0 \supsetneqq a, b \supsetneqq I$ in $\mathbb{M}_{2}$ be an absolutely compatible pair. Suppose $n(a) \neq 0$ or $s(a) \neq 0$. Then $a$ and $b$ commute and there exists a rank one projection $p$ in $\mathbb{M}_{2}$ such that one of the following three cases arises:

(1) $a=p$ and $b=\lambda p+\mu(I-p)$ for some $\lambda, \mu \in[0,1]$ with $0<\lambda+\mu<2$;

(2) $a=p+t(I-p)$ and $b=\lambda p+\mu(I-p)$ for some $t, \lambda \in[0,1), \mu \in\{0,1\}$; 
(3) $a=t p$ and $b=\lambda p+\mu(I-p)$ for some $t \in(0,1), \lambda \in\{0,1\}, \mu \in[0,1]$.

Proof. Suppose first that $s(a) \neq 0$. In this case, there exists a rank one projection $p=s(a)$ in $\mathbb{M}_{2}$ such that $a=p+t(I-p)$ for some $t \in[0,1)$ with $I-p \leq n(a)+r(e(a))$ and $I-p$ is a rank one projection. It follows that either $n(a)=0$ or $r(e(a))=0$. If $n(a) \neq 0$, then $a=p=s(a)$, and by applying Theorem 2.6 we deduce the existence of $\lambda, \mu \in[0,1]$ with $0<\lambda+\mu<2$ such that $b=\lambda s(a)+\mu n(a)$. If $e(a) \neq 0$, then $I-p=r(e(a))$ and Theorem 2.6 implies that statement (2) holds.

Suppose finally that $n(a) \neq 0$. The hypotheses imply that either $s(a)=0$ or $r(e(a))=0$. The case $e(a)=0$ (equivalently, $a=s(a) \neq 0$ ) has been discussed above. If $n(a)=0$, there exists $t \in(0,1)$ satisfying $a=t p$, where $p$ is a rank one projection. Theorem 2.6 implies that $b=\alpha p+\beta(I-p)$ for some $\alpha, \beta \in[0,1]$. Since $\alpha p \triangle t p$ and $t \neq 0,1$, we deduce that $\alpha \in\{0,1\}$.

We shall deal next with non-commuting pairs in $\mathbb{M}_{2}$. Let $0 \leq a, b \leq I$ be a non-commuting, absolutely compatible pair in $\mathbb{M}_{2}$. In this case, $a b \neq 0$. The case in which $n(a) \neq 0$ or $s(a) \neq 0$ has been treated in Proposition 3.2. We can thus assume, without any loss of generality, that $0 \supsetneqq a, b \varsubsetneqq I$ are absolutely compatible, $a b \neq 0$ and $n(a)=s(a)=0=n(b)=s(b)$. Henceforth, given $a \in \mathbb{M}_{n}$, the symbol trace $(a)$ will denote the (non-normalized) trace of $a$.

Theorem 3.3. Let $0 \supsetneqq a, b \supsetneqq I$ in $\mathbb{M}_{2}$. Then $a$ and $b$ are absolutely compatible with $a b \neq b a$ and $n(a)=s(a)=0=n(b)=s(b)$ if, and only if, the following three properties hold:

(1) $\operatorname{det}(a)>0, \operatorname{det}(b)>0$;

(2) $\operatorname{trace}(a)=1=\operatorname{trace}(b)$;

(3) $\operatorname{det}(a \circ b)=0$.

Proof. By hypothesis $n(a)=n(b)=s(a)=s(b)=0$. Accordingly to the notation and conclusions in Theorem 2.10, we also know that $s\left(b_{2}\right)=0=n\left(b_{2}\right)$. Therefore, by the just quoted theorem (see also Remark 2.12), we can assume, up to an appropriate representation, that $a=\left(\begin{array}{ll}\alpha_{11} & \alpha_{12} \\ \overline{\alpha_{12}} & \alpha_{22}\end{array}\right)$, and $b=\left(\begin{array}{cc}\beta_{11} & -\alpha_{12} \\ -\overline{\alpha_{12}} & \beta_{22}\end{array}\right)$, where $\alpha_{11}, \alpha_{22} \in \mathbb{R}_{0}^{+}, \alpha_{12} \in \mathbb{C}$, and $\operatorname{det}(a), \operatorname{det}(b)>0$ (the latter because $n(a)=$ $n(b)=0)$. We also know from Theorem 2.2 that the following identities hold

(1) $\left|\overline{\alpha_{12}}\right|^{2}=\left(1-\alpha_{11}\right)\left(1-\beta_{11}\right)$;

(2) $\left|\alpha_{12}\right|^{2}=\alpha_{22} \beta_{22}$;

(3) $\alpha_{12}=\left(\alpha_{11}+\alpha_{22}\right) \alpha_{12}=\left(\beta_{11}+\beta_{22}\right) \alpha_{12}$.

The case $\alpha_{12}=0$ is impossible because $a$ and $b$ do not commute. Therefore $\alpha_{11}+\alpha_{22}=\beta_{11}+\beta_{22}=1$. It is not hard to check that, in this case,

$$
a \circ b=\left(\begin{array}{cc}
\alpha_{11} \beta_{11}-\left|\alpha_{12}\right|^{2} & 0 \\
0 & \alpha_{22} \beta_{22}-\left|\alpha_{12}\right|^{2}
\end{array}\right)=\left(\begin{array}{cc}
\alpha_{11} \beta_{11}-\left|\alpha_{12}\right|^{2} & 0 \\
0 & 0
\end{array}\right),
$$

and thus, $\operatorname{det}(a \circ b)=0$.

Now let us assume that $a$ and $b$ satisfy (1) - (3). Since $a \circ b$ is a hermitian matrix with $\operatorname{det}(a \circ b)=0$, then there exists a rank one projection $p \in \mathbb{M}_{2}$ such that $a \circ b=\lambda p$ for some $\lambda \in[0,1]$. Find a unitary $u$ such that $u^{*} p u=\left(\begin{array}{ll}1 & 0 \\ 0 & 0\end{array}\right)$. As $\operatorname{trace}(a)=1=\operatorname{trace}(b)$, we have $a_{u}=u^{*} a u=\left(\begin{array}{cc}t & \alpha \\ \bar{\alpha} & 1-t\end{array}\right)$ and $b_{u}=u^{*} b u=$ 
$\left(\begin{array}{cc}s & \beta \\ \bar{\beta} & 1-s\end{array}\right)$. Having in mind that $a_{u} \circ b_{u}=\left(\begin{array}{cc}\lambda & 0 \\ 0 & 0\end{array}\right)$, we get $\alpha+\beta=0$ and $\alpha \bar{\beta}+\bar{\alpha} \beta+2(1-t)(1-s)=0$. Thus, $\beta=-\alpha$ and $|\alpha|^{2}=(1-t)(1-s)$. Theorem 2.2 implies that $a_{u} \triangle b_{u}$ and consequently $a \triangle b$.

We shall next show that $n(a)=s(a)=0=n(b)=s(b)$. Clearly $n(a)=0$ because $\operatorname{det}(a)>0$. If $s(a) \neq 0$, then there exists a rank one projection $q \in \mathbb{M}_{2}$ such that $q \leq a$. In this case, $a=q+t(1-q)$ for some $t \in[0,1]$. The condition $\operatorname{trace}(a)=1$ implies that $t=0$, and hence, $\operatorname{det}(a)=0$, which is impossible. We can similarly prove that $0=n(b)=s(b)$.

Finally, if $a b=b a$, then $a \circ b=a b=b a$ and $0=\operatorname{det}(a \circ b)=\operatorname{det}(a b)=\operatorname{det}(a) \operatorname{det}(b)>0$, which is impossible. We have therefore shown that $a b \neq b a$, and in particular $a b \neq 0$.

Let us comment some more concrete conclusions and geometric interpretations.

REMARK 3.4. Let $0 \supsetneqq a, b \supsetneqq I$ in $\mathbb{M}_{2}$ with $a \triangle b$ and $a b \neq b a$. By Theorem 3.3, there exists $t \in[0,1]$ and $\alpha \in \mathbb{C}$, such that $\operatorname{det}(a)=\operatorname{det}\left(\begin{array}{cc}t & \alpha \\ \bar{\alpha} & 1-t\end{array}\right)=t(1-t)-|\alpha|^{2} \leq \frac{1}{4}-|\alpha|^{2} \leq \frac{1}{4} \cdot \operatorname{Similarly}, \operatorname{det}(b) \leq \frac{1}{4}$. Furthermore, $\operatorname{det}(a)=\frac{1}{4}$ if, and only if, $t=\frac{1}{2}$ and $\alpha=0$, or equivalently, $a=\frac{1}{2} I$, which is impossible because $a b \neq b a$. We have therefore shown that $\operatorname{det}(a), \operatorname{det}(b)<\frac{1}{4}$.

We note that the matrices $a$ and $b$ belong to the set

$$
\begin{aligned}
\mathcal{S} & =\left\{c \in \mathbb{M}_{2}: 0 \leq c \leq I, \operatorname{trace}(c)=1,0<\operatorname{det}(c)<\frac{1}{4}\right\} \\
& =\left\{\left(\begin{array}{cc}
t & \alpha \\
\bar{\alpha} & 1-t
\end{array}\right): t \in(0,1), \alpha \in \mathbb{C} \text { and }|\alpha|^{2}<t(1-t)\right\} \backslash\left\{\frac{1}{2} I\right\} .
\end{aligned}
$$

We observe that every element $c \in \mathcal{S}$ satisfies $n(c)=s(c)=0$.

Let $a$ be an element in $\mathcal{S}$. We can now conclude that, up to an appropriate ${ }^{*}$-isomorphism, we can determine the set of absolutely compatible elements in $\mathcal{S}$.

THEOREM 3.5. Let $a$ and $b$ be two matrices in the set $\mathcal{S} \subset \mathbb{M}_{2}$. Then $a$ is absolutely compatible with $b$ if, and only if, there exists a unitary $u \in \mathbb{M}_{2}$ such that $u^{*} a u=\left(\begin{array}{cc}t & \alpha \\ \bar{\alpha} & 1-t\end{array}\right)$ and $u^{*} b u=\left(\begin{array}{cc}s \\ \bar{\beta} & 1-s\end{array}\right)$, where $\beta=-\alpha \neq 0$ and $s=\frac{|\alpha|^{2}}{t}$ or $s=1-\frac{|\alpha|^{2}}{1-t}$ with $s, t \in(0,1)$ and $|\alpha|^{2}<t(1-t)$.

Proof. We begin with some observations. By the hypotheses, $n(a)=s(a)=0=n(b)=s(b)$. Actually, $\operatorname{det}(a)>0, \operatorname{det}(b)>0$ and $\operatorname{trace}(a)=1=\operatorname{trace}(b)$.

Suppose $a \triangle b$. Since $a$ and $b$ are invertible elements in $\mathbb{M}_{2}$, it then follows that $a$ and $b$ are not orthogonal, and hence, $a \circ b \neq 0$ (Theorem 2.2). We observe that in $\mathbb{M}_{2}$ there are no more than two mutually orthogonal rank one projections. Theorem 3.3 implies that $\operatorname{det}(a \circ b)=0$, and hence, the range projection of $a \circ b$ is a rank one projection. Having in mind that for each unitary $u \in \mathbb{M}_{2}$ we have $u^{*} \mathcal{S} u=\mathcal{S}$, by applying Theorem 2.2, we can find a unitary element $u \in \mathbb{M}_{2}$ such that $a_{u}=u^{*} a u=\left(\begin{array}{cc}t & \alpha \\ \bar{\alpha} & 1-t\end{array}\right) \in \mathcal{S}$ and $b_{u}=u^{*} b u=\left(\begin{array}{cc}s & -\alpha \\ -\bar{\alpha} & 1-s\end{array}\right) \in \mathcal{S}$, with $|\alpha|^{2}<\min \{t(1-t), s(1-s)\}$.

In this case, $a_{u} b_{u}=b_{u} a_{u}$ if, and only if, $\alpha=0$ or $s+t=1$. The second case is impossible because if 
$s+t=1$ we would have $b_{u}=I-a_{u}$, and thus,

$$
I=\left|b_{u}-a_{u}\right|+\left|I-b_{u}-a_{u}\right|=\left|b_{u}-a_{u}\right|=\left|I-2 a_{u}\right|=\lambda_{2} q_{1}+\left|\lambda_{1}\right| q_{2} \neq I,
$$

where $q_{1}$ is a rank one projection, $q_{2}=I-q_{1}$, and

$$
1>\lambda_{2}=\sqrt{1-4\left(t(1-t)-|\alpha|^{2}\right)}>0>\lambda_{1}=-\lambda_{2}>-1
$$

are the eigenvalues of $I-2 a_{u}$. The case $\alpha=0$ also is impossible because $s, t \in(0,1) \backslash\left\{\frac{1}{2}\right\}, a_{u} \triangle b_{u}$, and $a_{u} b_{u}=b_{u} a_{u}$.

We have deduced that $a b \neq b a$ (equivalently, $a_{u} b_{u} \neq b_{u} a_{u}$ ). Theorem 3.3 implies that $a \triangle b$ (equivalently, $\left.a_{u} \triangle b_{u}\right)$ if, and only if, $\operatorname{det}(a \circ b)=0$ (equivalently, $\left.\operatorname{det}\left(a_{u} \circ b_{u}\right)=0\right)$. It can be easily seen that $\operatorname{det}\left(a_{u} \circ b_{u}\right)=$ $\left(s t-|\alpha|^{2}\right)\left((1-s)(1-t)-|\alpha|^{2}\right)=0$, and thus, st $=|\alpha|^{2}$ or $(1-s)(1-t)=|\alpha|^{2}$.

On the other hand, since $|\alpha|^{2}<t(1-t)<\frac{1}{4}$, it can be easily seen that $t>|\alpha|^{2}$ or $(1-t)>|\alpha|^{2}$. If $t>|\alpha|^{2}$ and $(1-t) \leq|\alpha|^{2}$, we deduce that $s=\frac{|\alpha|^{2}}{t} \in(0,1)$ and $b_{u}=\left(\begin{array}{cc}\frac{|\alpha|^{2}}{t} & -\alpha \\ -\bar{\alpha} & 1-\frac{|\alpha|^{2}}{t}\end{array}\right)$. If $t \leq|\alpha|^{2}$ and $(1-t)>|\alpha|^{2}$, we deduce that $s=1-\frac{|\alpha|^{2}}{1-t} \in(0,1)$ and $b_{u}=\left(\begin{array}{cc}1-\frac{|\alpha|^{2}}{1-t} & -\alpha \\ -\bar{\alpha} & \frac{|\alpha|^{2}}{1-t}\end{array}\right)$. If $t>|\alpha|^{2}$ and $(1-t)>|\alpha|^{2}$ both solutions are possible.

Finally, if there exists a unitary $u \in \mathbb{M}_{2}$ such that $u^{*} a u=\left(\begin{array}{cc}t & \alpha \\ \bar{\alpha} & 1-t\end{array}\right)$ and $u^{*} b u=\left(\begin{array}{cc}s & \beta \\ \bar{\beta} & 1-s\end{array}\right)$, in the conditions of the theorem, it is easy to check that $a b \neq b a, \operatorname{det}(a)>0, \operatorname{det}(b)>0, \operatorname{trace}(a)=1=\operatorname{trace}(b)$, and $\operatorname{det}(a \circ b)=0$. Theorem 3.3 assures that $a \triangle b$.

The set $\mathcal{S}$ can be identified with the punctured open ball in $\mathbb{R}^{3}$ given by

$$
\begin{aligned}
\stackrel{\circ}{\mathcal{B}} & =\left\{(t, \Re \mathrm{e}(\alpha), \Im \mathrm{m}(\alpha)): 0<\left(t-\frac{1}{2}\right)^{2}+\Re \mathrm{e}(\alpha)^{2}+\Im \mathrm{m}(\alpha)^{2}<\frac{1}{4}\right\} \\
& =\operatorname{int}\left(\mathcal{B}_{\mathbb{R}^{3}}\left(\left(\frac{1}{2}, 0,0\right), \frac{1}{2}\right)\right) \backslash\left\{\left(\frac{1}{2}, 0,0\right)\right\} .
\end{aligned}
$$

Fix $a=\left(\begin{array}{cc}t & \alpha \\ \bar{\alpha} & 1-t\end{array}\right) \in \mathcal{S}$ and let us consider the corresponding point $\widetilde{a}=(t, \Re \mathrm{e}(\alpha), \Im \mathrm{m}(\alpha))$ in $\stackrel{\circ}{\mathcal{B}}$. Then $a^{\prime}:=I-a \in \mathcal{S}$ corresponds to the point $\widetilde{a^{\prime}}=(1-t,-\Re \mathrm{e}(\alpha),-\Im \mathrm{m}(\alpha)) \in \mathcal{B}$.

Let $\lambda_{1} \geq \lambda_{2} \geq 0$ be the eigenvalues of $a$. Then $1>\lambda_{1}>\lambda_{2}>0$ with $\lambda_{1}+\lambda_{2}=1$. Consider $p_{a}=$ $\frac{1}{\lambda_{1}-\lambda_{2}}\left(\begin{array}{cc}t-\lambda_{2} & \alpha \\ \bar{\alpha} & \lambda_{1}-t\end{array}\right)$. It is not hard to check that $p_{a}$ is a rank one projection, and the corresponding point $\widetilde{p_{a}}=\frac{1}{\lambda_{1}-\lambda_{2}}\left(t-\lambda_{2}, \Re \mathrm{e}(\alpha), \Im \mathrm{m}(\alpha)\right) \in \mathbb{R}^{3}$ lies in the outer boundary of $\stackrel{\circ}{\mathcal{B}}$ (that is, $\left\|\widetilde{p_{a}}-(1 / 2,0,0)\right\|_{2}^{2}=$ $1 / 4$ in $\left.\mathbb{R}^{3}\right)$. Further, for the rank one projection $1-p_{a}=p_{a}^{\prime}=\frac{1}{\lambda_{1}-\lambda_{2}}\left(\begin{array}{cc}\lambda_{1}-t & -\alpha \\ -\bar{\alpha} & t-\lambda_{2}\end{array}\right)$, its corresponding point $\widetilde{p_{a}^{\prime}}=\frac{1}{\lambda_{1}-\lambda_{2}}\left(\lambda_{1}-t,-\Re \mathrm{e}(\alpha),-\Im \mathrm{m}(\alpha)\right)$ also lies in the outer boundary of $\stackrel{\circ}{\mathcal{B}}$. 
REMARK 3.6. Let $a$ and $b$ be two matrices in the set $\mathcal{S} \subset \mathbb{M}_{2}$. Suppose $a$ is absolutely compatible with $b$. Suppose first that there exists a unitary $u \in \mathbb{M}_{2}$ such that $a_{u}=u^{*} a u=\left(\begin{array}{cc}t & \alpha \\ \bar{\alpha} & 1-t\end{array}\right)$ and $b_{u}=u^{*} b u=$ $\left(\begin{array}{cc}\frac{|\alpha|^{2}}{t} & -\alpha \\ -\bar{\alpha} & 1-\frac{|\alpha|^{2}}{t}\end{array}\right)$, whose coordinates in $\mathbb{R}^{3}$ are $\widetilde{a_{u}}=(t, \Re \mathrm{e}(\alpha), \Im \mathrm{m}(\alpha))$ and $\widetilde{b_{u}}=\left(\frac{|\alpha|^{2}}{t},-\Re \mathrm{e}(\alpha),-\Im \mathrm{m}(\alpha)\right)$.

Consider the spheroid

$$
\mathcal{E}_{a_{u}}=\left\{x \in \mathbb{R}^{3}: d_{2}\left(x, \widetilde{a_{u}}\right)+d_{2}\left(x, \widetilde{a_{u}^{\prime}}\right)=1\right\}
$$

It is not hard to check that

$$
\begin{aligned}
\left\|\widetilde{b_{u}}-\widetilde{a_{u}}\right\|_{2}+\left\|\widetilde{b_{u}}-\widetilde{a_{u}^{\prime}}\right\|_{2} & =\sqrt{\left(t-\frac{|\alpha|^{2}}{t}\right)^{2}+4 \Re \mathrm{e}(\alpha)^{2}+4 \Im \mathrm{m}(\alpha)^{2}}+\left|1-t-\frac{|\alpha|^{2}}{t}\right| \\
& =\sqrt{\left(t-\frac{|\alpha|^{2}}{t}\right)^{2}+4|\alpha|^{2}}+\left|1-t-\frac{|\alpha|^{2}}{t}\right| \\
& =\sqrt{\left(t+\frac{|\alpha|^{2}}{t}\right)^{2}}+\left|1-t-\frac{|\alpha|^{2}}{t}\right| \quad \quad \quad\left(\text { since } t(1-t)>|\alpha|^{2}\right) \\
& =t+\frac{|\alpha|^{2}}{t}+1-t-\frac{|\alpha|^{2}}{t}=1
\end{aligned}
$$

that is $\widetilde{b_{u}} \in \mathcal{E}_{a_{u}}$. Similarly, for $b_{u}=\left(\begin{array}{cc}1-\frac{|\alpha|^{2}}{1-t} & -\alpha \\ -\bar{\alpha} & \frac{|\alpha|^{2}}{1-t}\end{array}\right)$, we can also show that $\widetilde{b_{u}} \in \mathcal{E}_{a_{u}}$.

Let us observe that $\widetilde{p_{a}}, \widetilde{p_{a}^{\prime}} \in \mathcal{E}_{a} \backslash \mathcal{S}$. Indeed, by applying that

$$
\lambda_{1}-\lambda_{2}=\sqrt{(2 t-1)^{2}+4 x^{2}+4 y^{2}},
$$

we can easily check that

$$
\left\|\widetilde{p_{a_{u}}}-\widetilde{a_{u}}\right\|_{2}+\left\|\widetilde{p_{a_{u}}}-\widetilde{a_{u}^{\prime}}\right\|_{2}=\frac{\|(2 t-1,2 x, 2 y)\|_{2}}{\lambda_{1}-\lambda_{2}}=1,
$$

and

$$
\left\|\widetilde{p_{a_{u}}^{\prime}}-\widetilde{a_{u}}\right\|_{2}+\left\|\widetilde{p_{a_{u}}^{\prime}}-\widetilde{a_{u}^{\prime}}\right\|_{2}=1
$$

The geometric interest is that the conclusion in the above remark actually is a pattern for absolutely compatible elements in $\mathbb{M}_{2}$.

Theorem 3.7. Let $a=\left(\begin{array}{cc}t & \alpha \\ \bar{\alpha} & 1-t\end{array}\right)$ and $b=\left(\begin{array}{cc}x & y+i z \\ y-i z & 1-x\end{array}\right)$ be two elements in $\mathcal{S}$. Then $a$ is absolutely compatible with $b$ if, and only if, the corresponding point $\widetilde{b}=(x, y, z)$ in $\mathbb{R}^{3}$ lies in $\mathcal{E}_{a} \backslash\left\{\widetilde{p_{a}}, \widetilde{p_{a}^{\prime}}\right\}$, with

$$
\mathcal{E}_{a}=\left\{x \in \mathbb{R}^{3}: d_{2}(x, \widetilde{a})+d_{2}\left(x, \widetilde{a^{\prime}}\right)=1\right\} .
$$

Proof. Let $|\alpha|=k$ and $\alpha=k e^{i \theta}$ for some $\theta \in[0,2 \pi]$. Assume that $a$ is absolutely compatible with $b$. Then by Theorem 3.3, $\operatorname{det}(a \circ b)=0$. Let $\beta=y+i z$. To simplify notation, we set $\varrho=\Re \mathrm{e}(\alpha \bar{\beta})=$ 
$k(y \cos \theta+z \sin \theta)$ By matrix calculations, we have

$$
\begin{aligned}
0 & =\operatorname{det}(a \circ b)=\operatorname{det}\left(\begin{array}{cc}
x t+\varrho & \frac{\alpha+\beta}{2} \\
\frac{\bar{\alpha}+\bar{\beta}}{2} & (1-x)(1-t)+\varrho
\end{array}\right) \\
& =(x t+\varrho)((1-x)(1-t)+\varrho)-\frac{|\alpha+\beta|^{2}}{4}
\end{aligned}
$$

if, and only if,

$$
y^{2}+z^{2}+k^{2}+2 \varrho=\left|y+i z+k e^{i \theta}\right|^{2}=|\alpha+\beta|^{2}=4(t x+\varrho)((1-t)(1-x)+\varrho),
$$

which is equivalent to the identity

$$
4 t(1-t) x^{2}+|\alpha|^{2}+|\beta|^{2}-4 \varrho^{2}+4(1-2 t) x \varrho-4 t(1-t) x-2(1-2 t) \varrho=0,
$$

or equivalently,

$$
\begin{aligned}
& 4 t(1-t) x^{2}+y^{2}+z^{2}-4 k^{2}(y \cos (\theta)+z \sin (\theta))^{2}+4 k(1-2 t) x(y \cos (\theta) \\
& +z \sin (\theta))-4 t(1-t) x-2 k(1-2 t)(y \cos (\theta)+z \sin (\theta))+k^{2}=0 .
\end{aligned}
$$

On the other hand, the equation

$$
\begin{aligned}
1 & =\|\widetilde{a}-\widetilde{b}\|_{2}+\left\|\widetilde{a^{\prime}}-\widetilde{b}\right\|_{2} \\
& =\|(t, \Re \mathrm{e}(\alpha), \Im \mathrm{m}(\alpha))-(x, y, z)\|_{2}+\|(1-t,-\Re \mathrm{e}(\alpha),-\Im \mathrm{m}(\alpha))-(x, y, z)\|_{2}
\end{aligned}
$$

can be rewritten in the form

$$
\begin{aligned}
& \sqrt{(1-t-x)^{2}+(-\Re \mathrm{e}(\alpha)-y)^{2}+(-\Im \mathrm{m}(\alpha)-z)^{2}} \\
& =1-\sqrt{(t-x)^{2}+(\Re \mathrm{e}(\alpha)-y)^{2}+(\Im \mathrm{m}(\alpha)-z)^{2}} .
\end{aligned}
$$

By squaring both sides and simplifying we get

$$
\sqrt{(t-x)^{2}+(\Re \mathrm{e}(\alpha)-y)^{2}+(\Im \mathrm{m}(\alpha)-z)^{2}}=t+x-2 t x-2 \Re \mathrm{e}(\alpha) y-2 \Im \mathrm{m}(\alpha) z .
$$

The left hand side coincides with $\sqrt{(t-x)^{2}+|\alpha-\beta|^{2}}$, while the right hand side equals to $(t+x)-2 t x-$ $2 \Re \mathrm{e}(\alpha \bar{\beta})=(t+x)-2 t x-2 \rho$. Now, by replacing both sides and squaring one more time we get

$$
\begin{gathered}
(t-x)^{2}+|\alpha|^{2}+|\beta|^{2}-2 \rho=(t-x)^{2}+|\alpha|^{2}+|\beta|^{2}-2 \Re \mathrm{e}(\alpha \bar{\beta})=(t-x)^{2}+|\alpha-\beta|^{2} \\
=(t+x)^{2}+4 t^{2} x^{2}+4 \rho^{2}-4(t+x) t x-4(t+x) \rho+8 t x \rho,
\end{gathered}
$$

which simplified yields the identity in (3.5).

We have proved that, under our hypotheses, $a$ is absolutely compatible with $b$ if, and only if, $\operatorname{det}(a \circ b)=0$ if, and only if, (3.5) holds if, and only if, $1=\|\widetilde{a}-\widetilde{b}\|_{2}+\left\|\widetilde{a^{\prime}}-\widetilde{b}\right\|_{2}$, equivalently, $\widetilde{b} \in \mathcal{E}_{a} \backslash\left\{\widetilde{p_{a}}, \widetilde{p_{a}^{\prime}}\right\}$.

An alternative approach, employed in later arguments, can be obtained by substituting in (3.6) $Y=$ $y \cos \theta+z \sin \theta$ and $Z=-y \sin \theta+z \cos \theta$. In this case, the commented equation transforms into

$$
4 t(1-t) x^{2}+Y^{2}+Z^{2}-4 k^{2} Y^{2}+4 k(1-2 t) x Y-4 t(1-t) x-2 k(1-2 t) Y+k^{2}=0 .
$$


Now, with a bit of patience, it is not hard to check that for $U=x \cos \phi-Y \sin \phi, V=x \sin \phi+Y \cos \phi$, $\cos \phi=\frac{1-2 t}{\sqrt{1-4 d}}, \sin \phi=\frac{2 k}{\sqrt{1-4 d}}$ and $d=\operatorname{det}(a)$, the previous equation is equivalent to

$$
4 d\left(U-\frac{1}{2} \cos \phi\right)^{2}+\left(V-\frac{1}{2} \sin \phi\right)^{2}+Z^{2}=d .
$$

Hence, (3.7), and therefore (3.5), represents a prolate spheroid with semi-major axis $\frac{1}{2}$, eccentricity $\sqrt{1-4 d}$. Further, we note that with respect to the coordinate system $(U, V, Z),(3.7)$ has centre at $\left(\frac{1}{2} \cos \phi, \frac{1}{2} \sin \phi, 0\right)$; foci at

$$
F_{1}\left(\frac{1}{2}\left(\cos \phi-\sqrt{1-4 d}, \frac{1}{2} \sin \phi, 0\right)\right)
$$

and

$$
F_{2}\left(\frac{1}{2}\left(\cos \phi+\sqrt{1-4 d}, \frac{1}{2} \sin \phi, 0\right)\right) ;
$$

and the extremities of the major axis at $E_{1}\left(\frac{1}{2}\left(\cos \phi-1, \frac{1}{2} \sin \phi, 0\right)\right)$ and $E_{2}\left(\frac{1}{2}\left(\cos \phi+1, \frac{1}{2} \sin \phi, 0\right)\right)$.

Transforming back to the coordinate system $(x, y, z)$, we may deduce that $(3.5)$ has the centre at $\left(\frac{1}{2}, 0,0\right)$, foci at $\widetilde{a}$, and $\widetilde{a^{\prime}}$ and the extremities of the major axis at $\widetilde{p_{a}}$ and $\widetilde{p_{a}^{\prime}}$.

REMARK 3.8. Let $a=\left(\begin{array}{cc}t & \alpha \\ \bar{\alpha} & 1-t\end{array}\right)$ be an element in $\mathcal{S}$. We keep the notation in the proof of Theorem 3.7. The spheroid $\mathcal{E}_{a}$ depends on the values of $t$ and $\alpha$. However, there is a geometric approach to reduce this dependence on the determinant of $a$. Namely, the director sphere of the prolate spheroid $\mathcal{E}_{a}$ given by (3.7) in the proof of the just mentioned theorem (i.e., the locus of the point of intersection of three tangent planes to this spheroid which are mutually at right angles, see [1, pages 86-87]) is defined by the equation

$$
\left(U-\frac{1}{2} \cos \phi\right)^{2}+\left(V-\frac{1}{2} \sin \phi\right)^{2}+Z^{2}=\frac{1}{4}+2 d .
$$

By transforming the previous equation into the coordinate system $(x, y, z)$ we have

$$
\left(x-\frac{1}{2}\right)^{2}+y^{2}+z^{2}=\frac{1}{4}+2 d,
$$

which only depends on $d=\operatorname{det}(a) \in\left(0, \frac{1}{4}\right)$. The union of all director spheres of the prolate spheroids $\mathcal{E}_{a}$ with $a$ running in the set $\mathcal{S}$ is precisely the (open) ring

$$
\left\{(x, y, z) \in \mathbb{R}^{3}: \frac{1}{4}<\left(x-\frac{1}{2}\right)^{2}+y^{2}+z^{2}<\frac{3}{4}\right\},
$$

whose inner boundary can be identified with

$$
\mathcal{P}_{1}\left(\mathbb{M}_{2}\right)=\left\{\left(\begin{array}{cc}
t & y+i z \\
y-i z & 1-t
\end{array}\right): \begin{array}{c}
t \in(0,1), y, z \in \mathbb{R} \\
y^{2}+z^{2}+\left(t-\frac{1}{2}\right)^{2}=\frac{1}{4}
\end{array}\right\}
$$

(which is defined as the set of all rank one projections in $\left.\mathbb{M}_{2}\right)$. Let us observe that $\mathcal{P}_{1}\left(\mathbb{M}_{2}\right)$ can be also identified with the outer boundary of the punctured open ball $\stackrel{\circ}{\mathcal{B}}$. Similarly, the centre of $(3.9)$ is $\left(\frac{1}{2}, 0,0\right)$ which is identified with $\frac{1}{2} I$. 
3.2. Absolute compatibility in $\mathbb{M}_{n}$. Our next goal is to study absolutely compatible pairs in $\mathbb{M}_{n}$.

TheOREM 3.9. Let $0 \leq a, b \leq I$ be elements in $\mathbb{M}_{n}$ with $n(a)=s(a)=0=n(b)=s(b)$. If $a$ is absolutely compatible with $b$, then $n$ is an even number, say $n=2 k$, and there exist $a_{1}, \ldots, a_{k}, b_{1}, \ldots, b_{k} \in \mathcal{S} \subseteq \mathbb{M}_{2}$ and a unitary $w \in \mathbb{M}_{n}$ with $a_{i} \triangle b_{i}$ for every $i=1, \ldots, k$ such that $a=w^{*}\left(a_{1} \oplus \cdots \oplus a_{k}\right) w$ and $b=w^{*}\left(b_{1} \oplus \cdots \oplus b_{k}\right) w$.

Proof. Let $\operatorname{rank}(a \circ b)=k$. By Theorem 2.2, for $p_{1}=r(a \circ b)$, there exist $a_{11}, b_{11} \in \mathbb{M}_{k}=p_{1} \mathbb{M}_{n} p_{1}$; $a_{22}, b_{22} \in \mathbb{M}_{n-k}=\left(I-p_{1}\right) \mathbb{M}_{n}\left(I-p_{1}\right)$ and $a_{12} \in \mathbb{M}_{k, n-k}$ with

$$
\begin{gathered}
a_{12} a_{12}^{*}=\left(p_{1}-a_{11}\right)\left(p_{1}-b_{11}\right), \quad a_{11} b_{11}=b_{11} a_{11}, \\
a_{12}^{*} a_{12}=a_{22} a_{22}=b_{22} a_{22}, \\
a_{12}=a_{11} a_{12}+a_{12} a_{22}=b_{11} a_{12}+a_{12} b_{22},
\end{gathered}
$$

such that $a=\left(\begin{array}{ll}a_{11} & a_{12} \\ a_{12}^{*} & a_{22}\end{array}\right)$ and $b=\left(\begin{array}{cc}b_{11} & -a_{12} \\ -a_{12}^{*} & b_{22}\end{array}\right)$. Since $a_{11}, a_{22}, b_{11}$, and $b_{22}$ are positive with $a_{11} b_{11}=b_{11} a_{11}$ and $a_{22} b_{22}=b_{22} a_{22}$, we can find unitaries $u_{1} \in \mathbb{M}_{k}$ and $u_{2} \in \mathbb{M}_{n-k}$ such that $u_{1}^{*} a_{11} u_{1}=D_{1}$, $u_{1}^{*} b_{11} u_{1}=E_{1}, u_{2}^{*} a_{22} u_{2}=D_{2}$ and $u_{2}^{*} b_{22} u_{2}=E_{2}$ are diagonal. Put $S_{12}=u_{1}^{*} a_{12} u_{2}$. Then for $u=u_{1} \oplus u_{2}$, we have

$$
a_{u}=u^{*} a u=\left(\begin{array}{cc}
D_{1} & S_{12} \\
S_{12}^{*} & D_{2}
\end{array}\right) \quad \text { and } \quad b_{u}=u^{*} b u=\left(\begin{array}{cc}
E_{1} & -S_{12} \\
-S_{12}^{*} & E_{2}
\end{array}\right)
$$

so that

$$
\begin{gathered}
S_{12} S_{12}^{*}=\left(p_{1}-D_{1}\right)\left(p_{1}-E_{1}\right) ; \\
S_{12}^{*} S_{12}=D_{2} E_{2} ; \\
S_{12}=D_{1} S_{12}+S_{12} D_{2}=E_{1} S_{12}+S_{12} E_{2} .
\end{gathered}
$$

Let $D_{1}=\operatorname{diag}\left(\alpha_{1}, \ldots, \alpha_{k}\right), D_{2}=\operatorname{diag}\left(\alpha_{k+1}, \ldots, \alpha_{n}\right), E_{1}=\operatorname{diag}\left(\beta_{1}, \ldots, \beta_{k}\right)$ and $E_{2}=\operatorname{diag}\left(\beta_{k+1}\right.$, $\left.\ldots, \beta_{n}\right)$. Since $n(a)=s(a)=0=n(b)=s(b)$, the same conclusion holds for $a_{u}$ and $b_{u}$. Thus, $\alpha_{i}, \beta_{j} \in(0,1)$, for $1 \leq i, j \leq n$. In particular, $\operatorname{rank}\left(S_{12} S_{12}^{*}\right)=k$ and $\operatorname{rank}\left(S_{12}^{*} S_{12}\right)=n-k$. Therefore,

$$
\max \{k, n-k\} \leq \operatorname{rank}\left(S_{12}\right) \leq \min \{k, n-k\} .
$$

In other words, $\operatorname{rank}\left(S_{12}\right)=k=n-k$, so that $n=2 k$.

Let us denote $S_{12}=\left(s_{i j}\right)$. Then by (3.10) and (3.11), we have

$$
\begin{gathered}
\sum_{j=1}^{k}\left|s_{i j}\right|^{2}=\left(1-\alpha_{i}\right)\left(1-\beta_{i}\right), \quad 1 \leq i \leq k \\
\sum_{i=1}^{k}\left|s_{i j}\right|^{2}=\alpha_{k+j} \beta_{k+j}, \quad 1 \leq j \leq k \\
\sum_{l=1}^{k} s_{i l} \bar{s}_{j l}=0, \quad i \neq j
\end{gathered}
$$




$$
\sum_{l=1}^{k} \bar{s}_{l i} s_{l j}=0, \quad i \neq j .
$$

By (3.12), we have

$$
s_{i j}=\left(\alpha_{i}+\alpha_{k+j}\right) s_{i j}=\left(\beta_{i}+\beta_{k+j}\right) s_{i j}, \quad 1 \leq i, j \leq k .
$$

Since $\operatorname{rank}\left(S_{12}\right)=k, \operatorname{det}\left(S_{12}\right) \neq 0$. Also by the Leibnitz formula for the determinant, we have

$$
\operatorname{det}\left(S_{12}\right)=\sum_{\sigma \in S_{k}} \operatorname{sgn}(\sigma) \Pi_{i=1}^{k} s_{\sigma(i) i}
$$

we note that $\Pi_{i=1}^{k} s_{\sigma(i) i} \neq 0$ for some permutation $\sigma \in S_{k}$. Thus, replacing $s_{12}$ by $P_{\sigma^{-1}} s_{12}$, and $D_{1}$ by $P_{\sigma^{-1}} D_{1} P_{\sigma}$, we may assume that $s_{i i} \neq 0$ for $1 \leq i \leq k$.

Now, by (3.13),

$$
\alpha_{i}+\alpha_{k+i}=1=\beta_{i}+\beta_{k+i} \quad 1 \leq i \leq k .
$$

Applying (3.14) to (3.13), we may deduce that

$$
\left(\alpha_{i}-\alpha_{j}\right) s_{i j}=0=\left(\beta_{i}-\beta_{j}\right) s_{i j}, \quad 1 \leq i, j \leq k .
$$

Applying (3.14), we further get that $D_{1}+D_{2}=I_{k}=E_{1}+E_{2}$. Thus, by (3.10) and (3.11), we have

$$
S_{12} S_{12}^{*}=S_{12}^{*} S_{12}
$$

Similarly, by (3.12), we deduce that

$$
S_{12} D_{1}=D_{1} S_{12} \quad \text { and } \quad S_{12} E_{1}=E_{1} S_{12} .
$$

Having in mind that $S_{12}$ is normal, there exists a unitary $v \in \mathbb{M}_{k}$ such that $v^{*} D_{1} v, v^{*} E_{1} v$ and $v^{*} S_{12} v$ are diagonal, say,

$$
\begin{aligned}
& D:=v^{*} D_{1} v=\operatorname{diag}\left(\lambda_{1}, \ldots, \lambda_{k}\right) \\
& E:=v^{*} E_{1} v=\operatorname{diag}\left(\mu_{1}, \ldots, \mu_{k}\right) \\
& S:=v^{*} S_{12} v=\operatorname{diag}\left(s_{1}, \ldots, s_{k}\right) .
\end{aligned}
$$

It follows that

$$
a_{0}:=(v \oplus v)^{*} u^{*} a u(v \oplus v)=\left(\begin{array}{cc}
D & S \\
S^{*} & I_{k}-D
\end{array}\right)
$$


and

$$
b_{0}:=(v \oplus v)^{*} u^{*} b u(v \oplus v)=\left(\begin{array}{cc}
E & -S \\
-S^{*} & I_{k}-E
\end{array}\right)
$$

with $a_{0} \triangle b_{0}$. We can find a suitable permutation $P \in \mathbb{M}_{n}$ such that

$$
P a_{0} P=\left(\begin{array}{cc}
\lambda_{1} & s_{1} \\
\bar{s}_{1} & 1-\lambda_{1}
\end{array}\right) \oplus \cdots \oplus\left(\begin{array}{cc}
\lambda_{k} & s_{k} \\
\bar{s}_{k} & 1-\lambda_{k}
\end{array}\right)
$$

and

$$
P b_{0} P=\left(\begin{array}{cc}
\mu_{1} & -s_{1} \\
-\bar{s}_{1} & 1-\mu_{1}
\end{array}\right) \oplus \cdots \oplus\left(\begin{array}{cc}
\mu_{k} & -s_{k} \\
-\bar{s}_{k} & 1-\mu_{k}
\end{array}\right),
$$

and by Lemma 2.5, we have

$$
\left(\begin{array}{cc}
\lambda_{i} & s_{i} \\
\bar{s}_{i} & 1-\lambda_{i}
\end{array}\right) \triangle\left(\begin{array}{cc}
\mu_{i} & -s_{i} \\
-\bar{s}_{i} & 1-\mu_{i}
\end{array}\right) \quad \text { for } 1 \leq i \leq k .
$$

Acknowledgments. We would like to acknowledge the anonymous reviewers who made extremely useful and constructive suggestions which improved the final version of this manuscript.

\section{REFERENCES}

[1] H.F. Baker. Principles of Geometry, Vol. 3, Solid Geometry (reprint of the 1923 original). Cambridge Library Collection, Cambridge University Press, Cambridge, 2010.

[2] M. Burgos, F.J. Fernández-Polo, J.J. Garcés, J. Martínez Moreno, and A.M. Peralta. Orthogonality preservers in $C^{*}$-algebras, JB*-algebras and JB*-triples. J. Math. Anal. Appl., 348:220-233, 2008.

[3] N.K. Jana, A.K. Karn, and A.M. Peralta. Contractive linear preservers of absolutely compatible pairs between $C^{*}-$ algebras. Rev. R. Acad. Cienc. Exactas Fís. Nat. Ser. A Mat. RACSAM, 113:2731-2744, 2019.

[4] R.V. Kadison and J.R. Ringrose. Fundamentals of the Theory of Operator Algebras, Vol. I, Elementary Theory. Academic Press, Inc., New York, 1983.

[5] S. Kakutani. Concrete representation of abstract (M)-spaces. Ann. of Math. (2), 42:994-1024, 1941.

[6] A.K. Karn. A p-theory of ordered normed spaces. Positivity, 14:441-458, 2010.

[7] A.K. Karn. Orthogonality in $\ell_{p}$-spaces and its bearing on ordered Banach spaces. Positivity, 18:223-234, 2014.

[8] A.K. Karn. Orthogonality in a $C^{*}$-algebra. Positivity, 20:607-620, 2016.

[9] A.K. Karn. Algebraic orthogonality and commuting projections in operator algebras. Acta Sci. Math. (Szeged), 84:323$353,2018$.

[10] J.-H. Liu, C.-Y. Chou, C.-J. Liao, and N.-C. Wong. Disjointness preservers of $A W^{*}$-algebras. Linear Algebra Appl., 552:71-84, 2018 .

[11] J.-H. Liu, C.-Y. Chou, C.-J. Liao, and N.-C. Wong. Linear disjointness preservers of operator algebras and related structures. Acta Sci. Math. (Szeged), 84:277-307, 2018.

[12] G.K. Pedersen. $C^{*}$-Algebras and their Automorphism Groups. London Mathematical Society Monographs, Vol. 14, Academic Press, Inc., London, 1979.

[13] M.H. Stone. A general theory of spectra. I. Proc. Nat. Acad. Sci. U.S.A., 26:280-283, 1940.

[14] M. Wolff. Disjointness preserving operators in $C^{*}$-algebras. Arch. Math. (Basel), 62:248-253, 1994.

[15] N.-C. Wong. Triple homomorphisms of $C^{*}$-algebras. Southeast Asian Bull. Math., 29:401-407, 2005. 